\title{
L'artisanat de la peinture à Pompéi
}

Missions septembre 2011, 2012 et 2013

Marie Tuffreau-Libre, Isabelle Brunie et Sébastien Daré

\section{OpenEdition \\ 12 Journals}

Édition électronique

URL : http://journals.openedition.org/cefr/1205

DOI : $10.4000 /$ cefr. 1205

ISSN : 2282-5703

Éditeur

École française de Rome

Référence électronique

Marie Tuffreau-Libre, Isabelle Brunie et Sébastien Daré, «L'artisanat de la peinture à Pompéi »,

Chronique des activités archéologiques de l'École française de Rome [En ligne], Les cités vésuviennes, mis en ligne le 11 juin 2014, consulté le 11 septembre 2019. URL : http://journals.openedition.org/ cefr/1205; DOI : 10.4000/cefr.1205

Ce document a été généré automatiquement le 11 septembre 2019.

(c) École française de Rome 


\section{L'artisanat de la peinture à Pompéi}

Missions septembre 2011, 2012 et 2013

Marie Tuffreau-Libre, Isabelle Brunie et Sébastien Daré

\section{NOTE DE L'AUTEUR}

Nous remercions vivement $\mathrm{M}^{\mathrm{me}}$ la Soprintendente T. E. Cinquantaquattro et la dottoressa Gr. Stefani, pour l'aide et l'intérêt qu'elles nous ont apportés.

Les fouilles de 2001 et 2002 ont pu être réalisées grâce aux collaborateurs suivants :

Jacqueline Cadalen-Lesieur, Christian Cribellier, Delphine Dixneuf, Raphaël Huchin, Guy Lintz, Gilles Prilaux, David Wild.

1 Depuis 2011, nous avons mené plusieurs missions d'étude à Pompéi (Campanie), afin de poursuivre les recherches engagées depuis 1996 sur les pots à couleurs découverts lors des fouilles anciennes et récentes dans les cités du Vésuve. Il s'agissait de dresser un inventaire de ces récipients utilisés pour le travail de la peinture, et d'étudier les contextes et les matériels associés, afin de définir exactement l'activité pratiquée: magasin et fabricant de couleurs, atelier de peintres ou peintres au travail. L'étude de ces contextes montrait leur concentration dans la partie orientale de la ville, et plusieurs ensembles de ces pots à couleurs apparaissaient liés à des travaux de peinture en cours en 79 ap. J.-C. La présence d'un mobilier particulièrement abondant dans une des maisons fouillée en 1952 (I.9.9) suggérait l'existence à cet endroit d'un atelier de peintres. Dans le cadre du programme sur l'artisanat du Centre Jean Bérard de Naples, nous avons repris des fouilles en 2001 et 2002 sur cette maison et le matériel trouvé dans les nombreuses fosses mises au jour a confirmé cette hypothèse. Les missions de septembre 2011 et 2012 ont eu pour objet de compléter l'inventaire des objets exhumés en 1952, effectué en 1999 et 2000, préalablement à la fouille, et cela dans la perspective d'une publication globale des données concernant cette maison. La monographie sur l'atelier I.9.9 est maintenant bien avancée et nous avons entamé en septembre 2013 le programme proposé l'année dernière, qui consiste à reprendre et à terminer l'inventaire exhaustif des pots à couleurs 
de Pompéi afin de publier une seconde monographie regroupant ces ensembles qui dessinent une image diversifiée de l'artisanat de la peinture et de la décoration à Pompéi.

2 Étalé sur trois ans de 2013 à 2015, ce travail consiste à reprendre la documentation considérable déjà réunie et à la compléter, à la fois par les pots à couleurs non encore inventoriés, notamment ceux d'Herculanum, très peu nombreux, et surtout par les inventaires d'objets associés qui nous permettent de déterminer avec précision l'activité en cours dans les maisons concernées. Le travail a été découpé en trois volets :

- Septembre 2013 : Inventaire des contextes de la Région I (ins. 6, 7, 9, 13, 18).

- Septembre 2014 : Inventaire des contextes des Régions II (ins. 1 et 2) et III (ins. 4).

- Septembre 2015 : Inventaire des contextes des Régions V (ins. 3) et IX (ins. 2, 7, 12).

3 L'étude de cette année a donc porté sur les contextes de la Région I (ins. 6, 7, 9, 13, 18). Ces derniers n'ont pas tous le même intérêt, d'une part en raison du caractère des objets qui y ont été trouvés, d'autre part parce que les fouilles anciennes livrent parfois peu d'informations sur les contextes et que certains objets ont disparu durant la deuxième guerre mondiale. Les recherches effectuées cette année ont été fructueuses; un grand nombre d'objets ont pu être dessinés et photographiés, et ce travail a été complété par une étude importante et minutieuse des archives (carnets de fouilles et rapports conservés à Pompéi).

\section{L'atelier de peintres (I.9.9)}

La maison I.9.9 se situe dans une rue latérale, à l'est de l'insula 9 de la région I. Elle fait partie d'un ensemble de maisons et boutiques, dans un quartier à vocation commerciale et artisanale qui s'organise autour de la rue de Castricio (fig. 1). 
Fig. 1 - Pompéi, Reg. I.9.9, rue latérale.

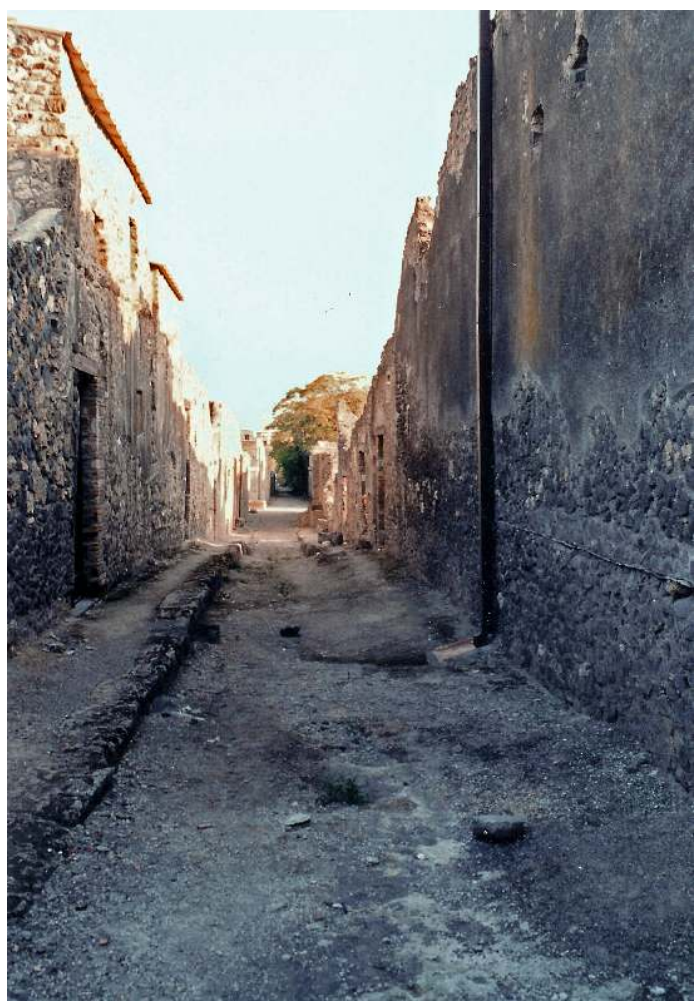

Photo Marie Tuffreau-Libre (sur concession du Ministero per i beni e le attività culturali - Soprintendenza per i beni archeologici di Napoli e Pompei. Toute reproduction, par quelque moyen que ce soit, reste interdite).

5 C'est une habitation de petite taille composée de 3 pièces desservies par un couloir qui aboutit à une arrière cour (fig. 2). 
Fig. 2 - Pompéi, plan de l'insula 9 (région I) et de la maison I.9.9 (fouilles 2001-2002).

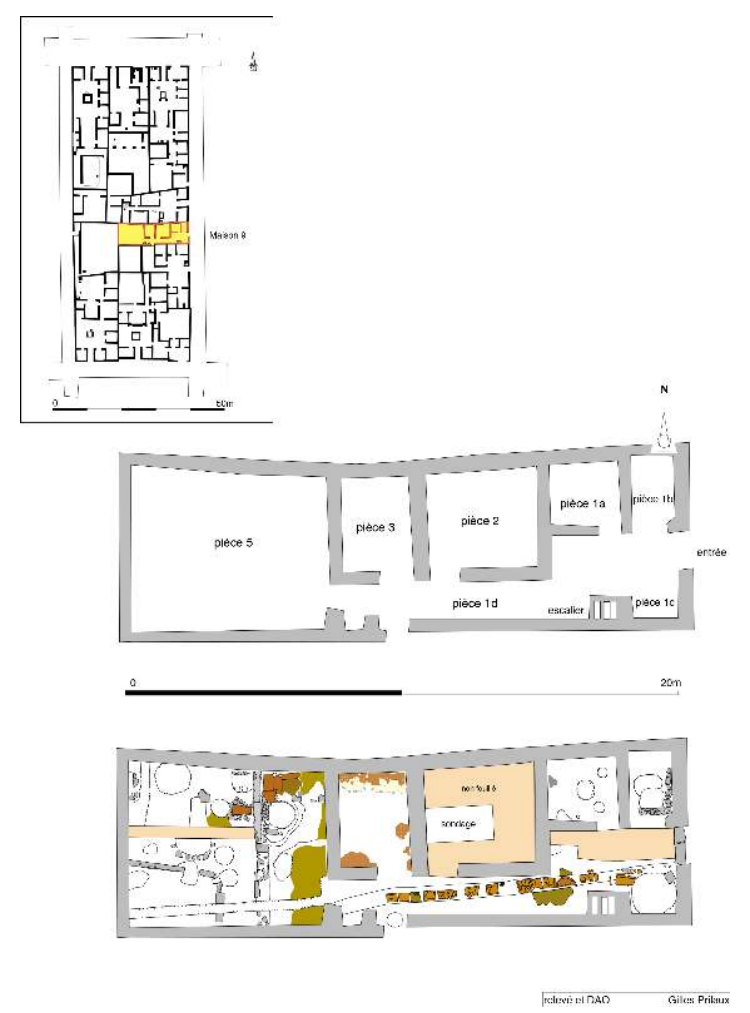

Relevé et DAO Gilles Prilaux (reproduction interdite).

6 À une période donnée, cette maison devait communiquer avec la maison 10, voisine, comme l'indique la présence de portes murées : l'une dans le couloir 4 , au bas de l'escalier et l'autre en haut de ce dernier. Par ailleurs, un puits situé à l'extrémité ouest du mur de limite des deux maisons devait être commun. Il semble que ce ne soit que dans les dernières phases de construction et d'occupation que les maisons 9 et 10 ont été définitivement séparées par le mur cloisonnant la cour arrière et la condamnation des différentes portes de communication. Cette séparation reflète peut-être une distinction plus marquée entre un espace dédié à l'habitation dans la maison 10 et un espace presque uniquement voué à des activités artisanales dans la maison 9. D'autres découvertes relatives aux pots à couleurs ont été faites dans ce quartier. La boutique de N. Fufidius Successus est proche et plusieurs trouvailles de pots à couleurs ont été faites dans la région I : en face de l'insula 8 , au $\mathrm{n}^{\circ} 2$ de l'insula 18, également dans les insulae 6 et 7 , dans l'insula 10, enfin dans l'insula XIII, au numéro 3. Toutes ces découvertes peuvent être liées à différentes activités artisanales, en rapport avec la décoration. Les fouilles de 1952 se déroulèrent en juillet et août, durant deux mois, et mirent au jour un matériel original composé, entre autres, d'environ 150 récipients contenant des couleurs. Une première série de 20 petits godets fut d'abord exhumée dans le couloir. À l'extrémité de ce couloir, dans l'angle, se trouvait une petite cuisine, située près du petit puits qui semble avoir été commun aux maisons 9 et 10 . C'est là qu'on a retrouvé un certain nombre d'ustensiles directement en rapport avec des fonctions culinaires: casseroles et vases en bronze, assiettes sigillées, carafes en verre.

7 Près de l'entrée de la maison, on découvrit ensuite une petite armoire en bois dont subsistait l'empreinte et qui contenait des objets variés destinés au travail de la peinture : 
des pilons de pierre de formes diverses dont un en forme de doigt, des plaques d'ardoise pour affûter les outils, des couteaux, lames et spatules en fer, un compas, des ustensiles à enduire, un fil à plomb, des poids de balance et la majeure partie des pots contenant des couleurs. Ce sont essentiellement des godets et des coupes de petites tailles, certains vides, d'autres contenant du noir, du blanc, du brun et divers tons de bleu, rouge, gris, jaune, orange, rose. Les fouilles récentes (2001 et 2002) ont confirmé la fonction de cet espace en révélant la présence de nombreuses fosses contenant des tessons présentant des restes de couleurs. Il s'agit donc d'un endroit où on prépare les couleurs, mais aussi où s'effectuent des travaux de décoration et de peinture (sur bois, sur terre cuite).

Parmi ces objets se trouve notamment une série de coquillages conservés au laboratoire d'archéologie de Pompéi dont l'usage dans cet atelier s'explique par la présence de couleur rose conservée dans l'un d'entre eux. Ils étaient utilisés comme godets pour mélanger de très petites quantités de couleurs, soit pour faire des essais de couleur, soit pour des retouches très limitées. Des recherches comparatives effectuées lors de la mission de 2013 ont révélé l'existence d'autres coquillages ayant contenu des couleurs et trouvés dans des contextes divers: l'un, contenant de la couleur rouge a été trouvé en 1934 près de la porte du Vésuve. Les deux autres, contenant respectivement des couleurs jaune et noire, sont sans provenance.

Des vases de formes diverses furent trouvés dans la pièce 2. Fouillée en juillet 1952, cette pièce a livré deux ensembles d'objets, situés dans les angles de la pièce, qui se différencient sensiblement de ceux trouvés dans l'armoire de la pièce $n^{\circ} 1$. Il s'agit essentiellement de vases de récupération, des gobelets à anse en paroi fine, contenant différents pigments de couleur blanche, marron, jaune et rouge, une grande olla remplie de pigment bleu et son couvercle. À cela s'ajoutent des pots à couleur de formes identiques à ceux découverts dans le couloir et dans l'armoire, mais de plus grande taille, contenant des pigments encore intacts de diverses couleurs ou des couleurs délayées. Il s'agit donc d'une pièce où l'on stockait les couleurs et les récipients de plus grande taille et où pouvaient s'effectuer différentes activités artistiques.

L'espace situé derrière la maison peut être considéré comme un espace ouvert. Des traces de charpentes, des sols bétonnés, les structures fouillées en 2001 et 2002 (bassin avec puits perdu servant d'exutoire) et le négatif d'une fondation nord-sud démontrent la présence d'appentis appuyés contre le mur ouest de la pièce 3 dévolus à des activités artisanales. Ces constructions légères, qui ne sont pas mentionnées sur le plan général de l'insula postérieur aux fouilles de 1952, furent détruites lors de l'éruption de 79. Plusieurs outils en fer, des broyeurs en pierre, des tessons portant des restes de couleurs confirment ici encore l'activité artisanale de l'habitation.

11 Parmi les objets retrouvés dans les fouilles récentes figure une palette (fig. 3) trouvée avec des fragments de coquille d'œuf suggérant la pratique de la tempera, peinture utilisant l'œuf pour lier les pigments. 
Fig. 3 - Pompéi, maison 1.9.9, fouilles 2001-2002, fond de céramique utilisé comme palette.

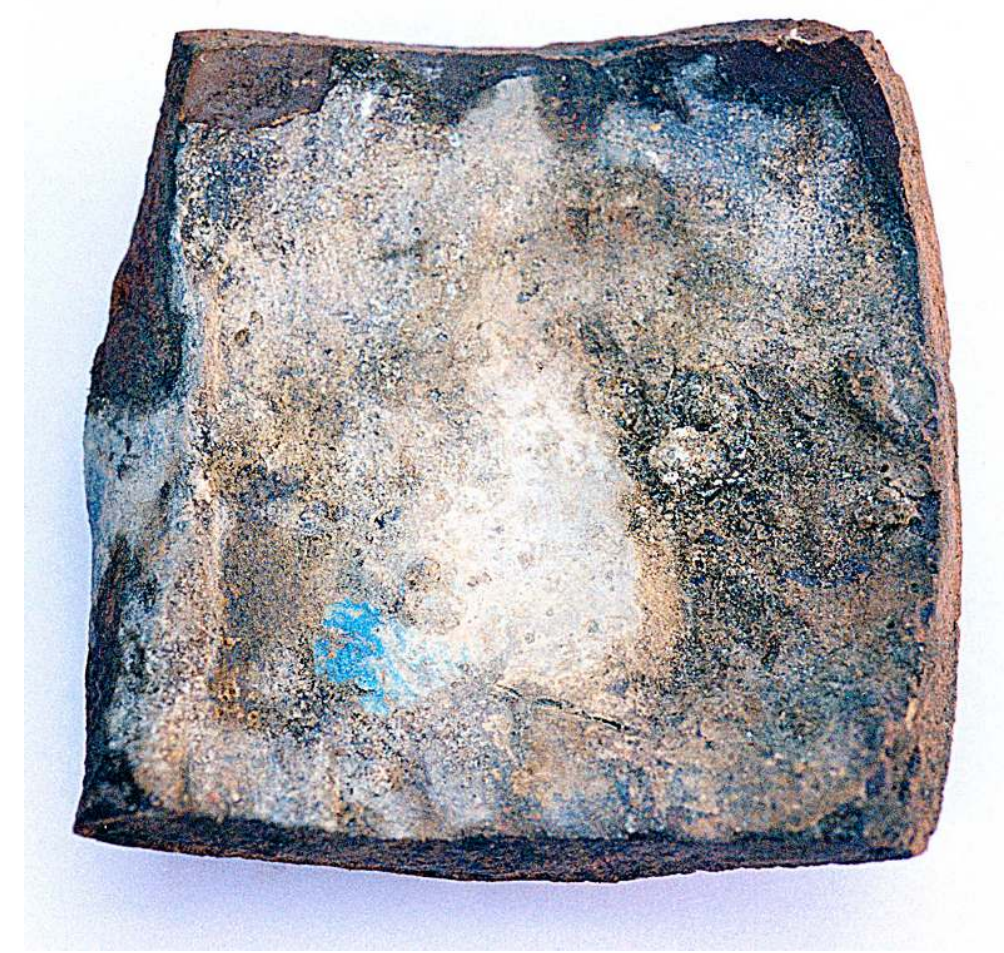

Photo Marie Tuffreau-Libre (sur concession du Ministero per i beni e le attività culturali - Soprintendenza per i beni archeologici di Napoli e Pompei. Toute reproduction, par quelque moyen que ce soit, reste interdite).

Ceci, ajouté à la présence des petits godets présents uniquement dans l'atelier et non ailleurs dans Pompéi, permet de conclure à la production dans cet atelier de peintures de chevalet. Des analyses ont été effectuées par François Delamare sur la palette, ainsi que sur les récipients contenant de la couleur trouvés en 1952. Un important ensemble de plaques en terre cuite polychromes a également été découvert dans le caniveau situé sous le couloir, ce qui suggère une certaine diversité dans les travaux de décoration des peintres de cet atelier, dont les débuts d'activité semblent remonter au $\mathrm{I}^{\mathrm{er}}$ siècle av. J.-C. 
Fig. 4 - Pompéi, maison I.9.9, fouilles 2001-2002 du caniveau sous le couloir, où ont été trouvées les plaques en terre cuite polychromes.

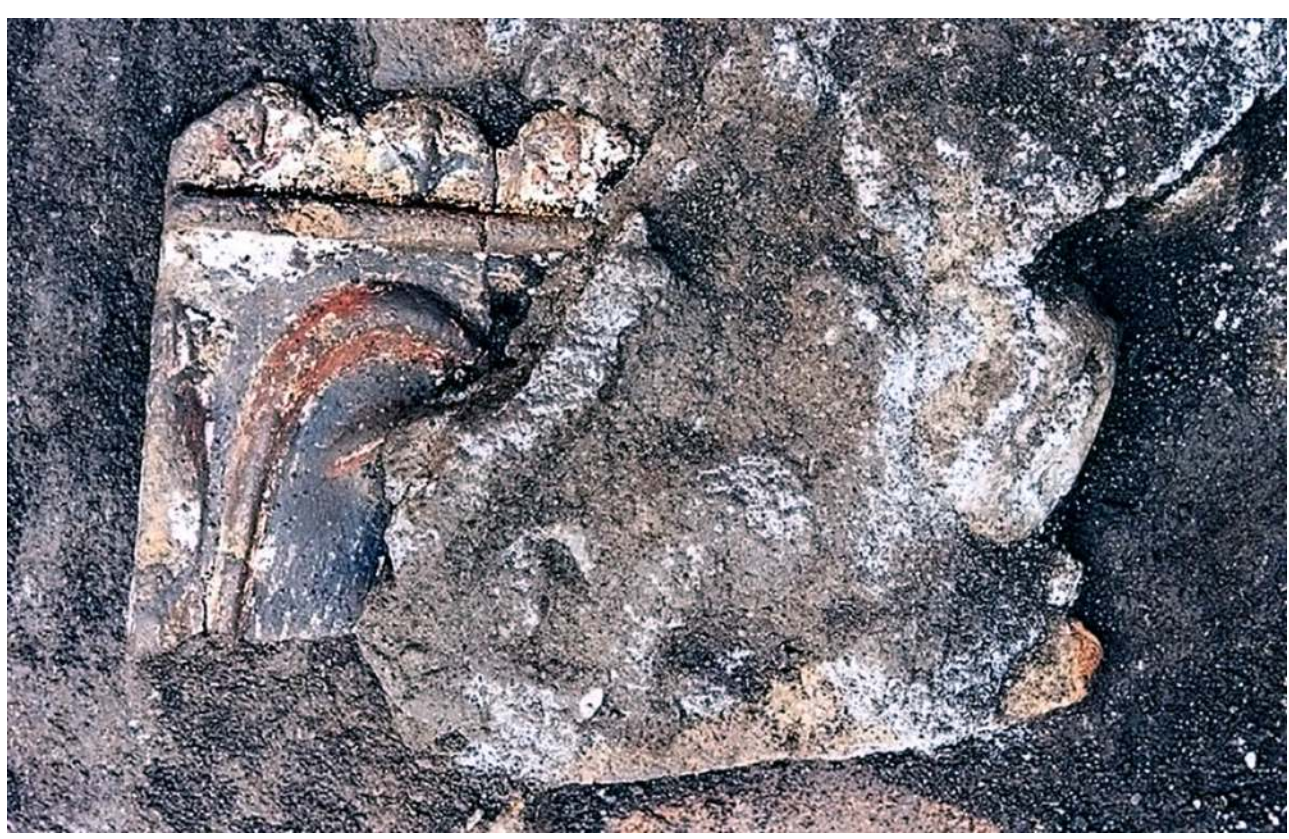

Photo Marie Tuffreau-Libre (sur concession du Ministero per i beni e le attività culturali - Soprintendenza per i beni archeologici di Napoli e Pompei. Toute reproduction, par quelque moyen que ce soit, reste interdite).

Cette boutique se situe tout près de la rue de Castricio et c'est dans ce quartier qu'a été mise en évidence par M. De Vos l'existence d'un atelier de peintres particulièrement actif officiant dans la ville pour la phase postérieure à 62 ap. J.-C. Il est fort probable que la maison I.9.9 soit le local abritant cet atelier de la rue de Castricio.

\section{La maison I.6.8}

Le premier contexte étudié en 2013 dans le cadre du nouveau programme a été celui de la maison I.6.8, endommagée lors des bombardements de 1943. Ce contexte a livré en 1912 un pot à couleur, une coupe contenant du pigment rouge. Le mobilier associé à cette découverte comprend relativement peu d'objets, des cruches en céramique commune, des fonds d'amphore, déposés au Granai del Foro, quelques objets en verre et en bronze, détruits lors des bombardements ou disparus.

\section{La maison du Cryptoportique}

15 Les contextes 2 et 16 de l'insula 6 correspondent à la maison du Cryptoportique, fouillée en 1913 et là encore, de nombreux objets ont disparu. Deux pots à couleurs proviennent de cette maison, probablement liés à des réfections ponctuelles. Il s'agit d'une petite coupe en céramique commune contenant des blocs de couleur rose et d'une autre contenant des blocs gris bleu. La disparition d'une part importante des objets lors des bombardements de 1943 ne permet pas d'aller plus loin dans l'interprétation. 


\section{L'Insula 16 (anciennement 18) (Région I)}

Dans une première étude publiée en 1999, nous avions mentionné un petit ensemble de vases de stockage remplis de pigments en blocs et en poudre provenant de l'insula 16.2. Nous avons donc commencé à étudier les objets de la maison 2, où avaient été trouvés les quatre pots à couleurs, mais l'étude des rapports de fouille ayant signalé que cette maison était liée aux deux maisons voisines (I.16.3 et I.16.4), et qu'apparemment toutes étaient en travaux de reconstruction et de réfection picturale au moment de l'éruption, nous avons demandé à compléter notre recherche avec l'étude de ces deux autres maisons. L'autorisation a été obtenue et nous avons pu photographier et dessiner les maisons et leur contenu. Les maisons 2, 3 et 4 ont été fouillées simultanément, essentiellement en 1957, et elles se révèlent effectivement toutes les trois en travaux de restauration et de peinture en 79 ap. J.-C. Cette insula comprend 7 maisons identifiées et de vastes espaces de jardin. Les fouilles n'ont pas été totalement achevées, mais les jardins ont été explorés par $\mathrm{M}^{\mathrm{me}}$ Jashemski.

Fig. 5 - Pompéi, plan de l'insula 16 (Région I) et des maisons étudiées : 2, 3, 4.

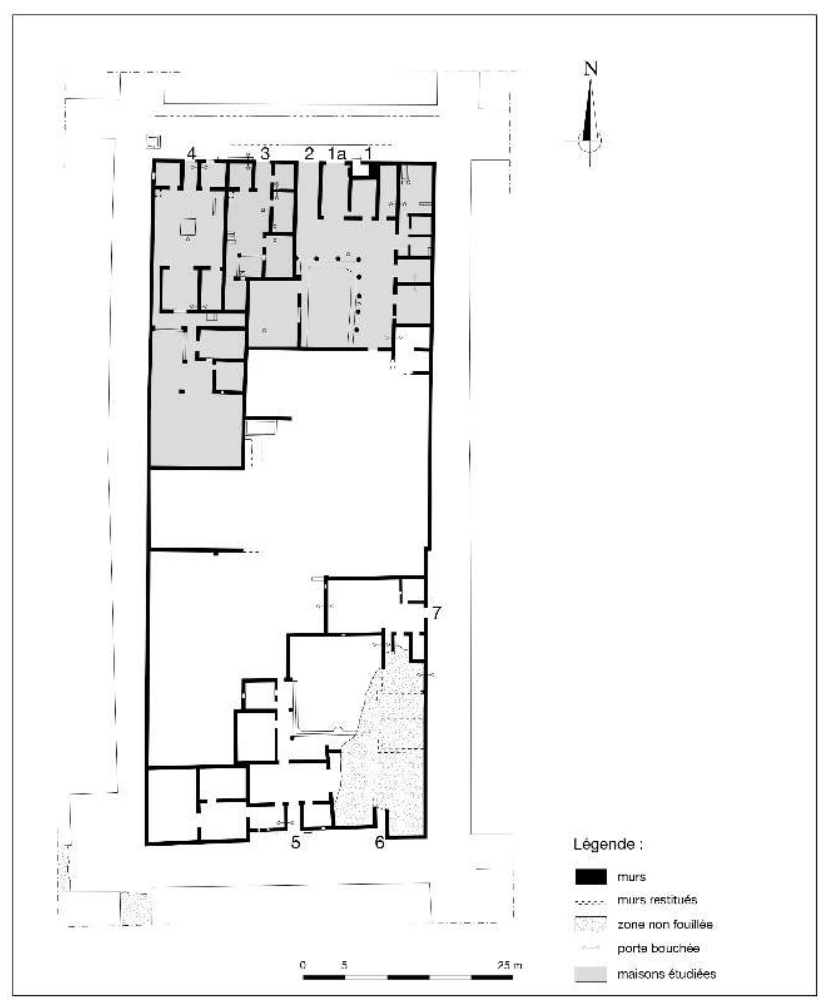

Plan Sébastien Daré (reproduction interdite).

Cet ensemble présente un grand intérêt pour les questions de technique picturale, ainsi que pour l'organisation du travail de la peinture à Pompéi. Il nous montre des peintres au travail simultanément dans trois maisons, à divers stades dans le travail de restauration. La maison 2, en cours de restructuration n'a pas encore été peinte, mais les travaux devaient s'effectuer prochainement, ce que suggèrent les pots de pigments stockés dans une des pièces. Les maisons 3 et 4 possèdent des laraires peints (fig. 6 et 7), peu de temps avant l'éruption et certaines de leurs pièces étaient également en cours de travaux. Il 
s'agit là d'une équipe d'artisans opérant dans plusieurs maisons, avec une coordination précise dans la succession des diverses opérations de maçonnerie et de peinture.

Fig. 6 - Pompéi, le laraire de la maison 3.

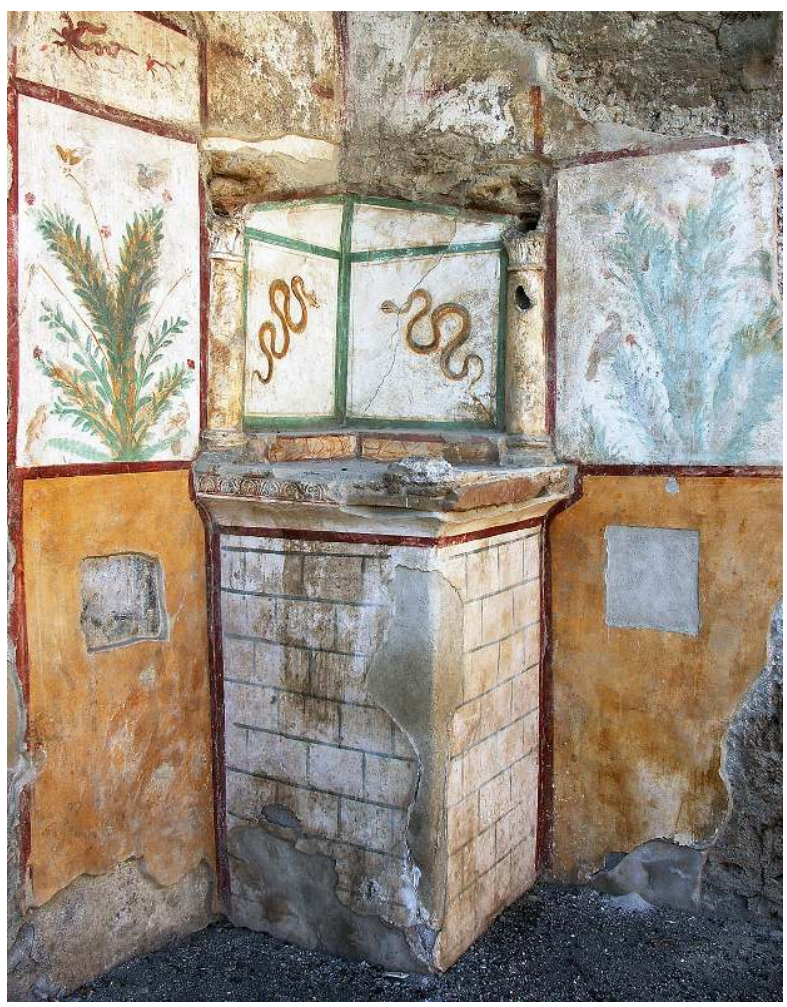

Photo Sébastien Daré (sur concession du Ministero per i beni e le attività culturali - Soprintendenza per i beni archeologici di Napoli e Pompei. Toute reproduction, par quelque moyen que ce soit, reste interdite). 
Fig. 7 - Pompéi, le laraire de la maison 4.

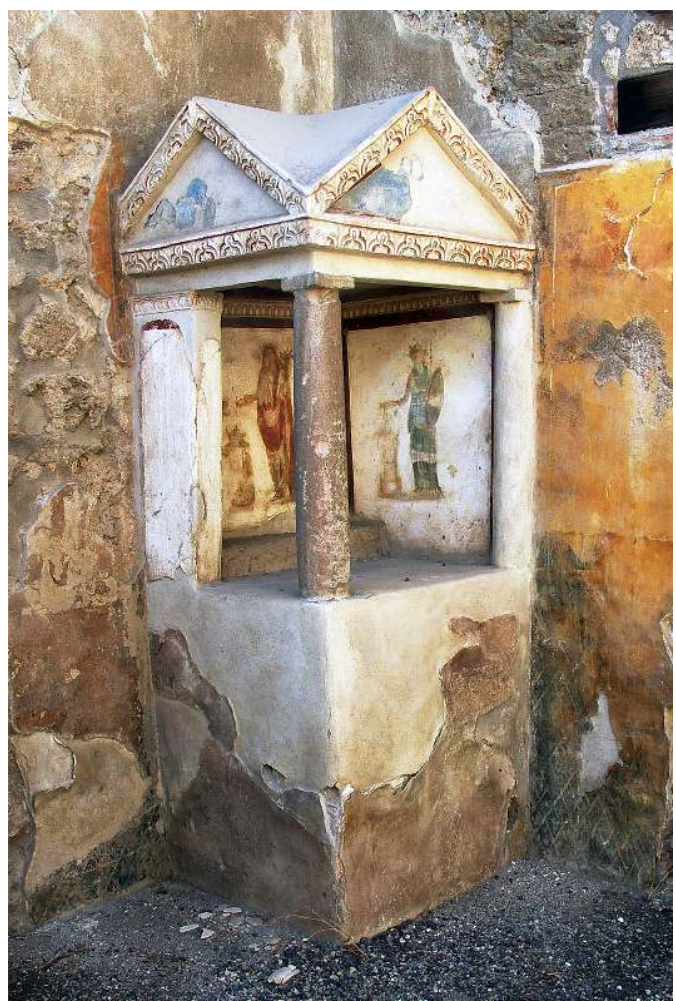

Photo Sébastien Daré (sur concession du Ministero per i beni e le attività culturali - Soprintendenza per i beni archeologici di Napoli e Pompei. Toute reproduction, par quelque moyen que ce soit, reste interdite).

Les peintures des maisons 3 et 4 , qui correspondent au IV style, pour la phase postérieure à 62 ap. J.-C. ?, ont été attribuées à l'atelier de peintre de la rue de Castricio, qui est donc selon toute vraisemblance, comme nous l'avons indiqué plus haut, localisé dans la maison I.9.9. L'ensemble des objets liés à la peinture est important. Les pots de stockage des couleurs, identiques à ceux trouvés dans l'atelier I.9.9 contiennent des nuances qui correspondent aux travaux en cours dans les maisons 3 et 4 . Certains récipients semblent être des objets récupérés et retaillés pour l'usage de la peinture. Les diverses lames en os, dont les usages sont multiples, portent des traces des couleurs utilisées pour les peintures en cours dans la maison 3, dans la pièce $3:$ du blanc, du jaune et surtout de l'orange. Il s'agit donc, pour ce contexte, d'un ensemble particulièrement intéressant, dont l'étude sera complétée et terminée lors de la prochaine mission, en septembre 2014. 


\section{BIBLIOGRAPHIE}

A. Barbet, M. Fuchs, M. Tuffreau-Libre, Les diverses utilisations des pigments et leurs contenants, dans Roman Wall Painting, Materials, Techniques, Analysis and Conservation, Actes du Colloque de Fribourg, Fribourg, 1997, p. 35-61.

A. Barbet, M. Tuffreau-Libre, Un ensemble de pots à couleurs à Pompéi, (II, I-9), dans Scienza e Societa, 25-27 novembre 1998, p. 255.

A. Barbet, M. Tuffreau-Libre, C. Coupry, Un ensemble de pots à couleurs à Pompéi, dans Rivista di Studii Pompeiani, 1999, p. 71-81.

Ph. Borgard, J.-P.Brun, M. Leguilloux, N. Monteix, M. Tuffreau-Libre, N. Cullin-Mingaud, Recherches sur les productions artisanales à Pompéi et à Herculanum, dans P. Giovanni Guzzo et M. P. Guidobaldi (éd.), Nuove ricercha archeologiche a Pompei ed Ercolano, Naples, 2005, p. 295-317.

M. De Vos, La bottega di pittori di via di Castricio, dans Pompei 1748-1980. I tempi della documentazione, Rome, 1981, p. 119-130.

W.Jashemski, The Gardens of Pompeii, Herculaneum, and the Villas Destroyed by Vesuvius, 2 vol. New Rochelle (NY), 1993.

M. Tuffreau-Libre, A. Barbet, Les pots à couleurs dans l'Antiquité romaine, dans SFECAG, Actes du Congrès du Mans, Marseille, 1997, p. 399-404.

M. Tuffreau-Libre, Les pots à couleur de Pompéi, premiers résultats, dans Rivista di Studii Pompeiani, 1999, p. 63-71.

M. Tuffreau-Libre (avec la collaboration de P. Arcelin, J. Cadalen, R. Huchin), La céramique de la maison I.9.9 à Pompéi, Italie, dans SFECAG, Actes du Congrès de Vallauris, Marseille, 2004, p. 315-327.

M. Tuffreau-Libre, Un atelier de peintres à Pompéi, Circulacion de temas y sistemas decorativos en la pintura mural Antigua, dans C. Guiral-Pelegrin (dir.), Actas del IX Congreso internacional de la Association internationale pour la peinture murale antique, Saragosse, 2007, p. 339-341.

A. Varone, L'organizzazione del lavoro di una bottega di decoratori : le evidenze dal récente scavo pompeiano lungo via dell'Abbondanza, dans MNIR, 54, 1995, p. 124-136.

\section{INDEX}

Mots-clés : atelier, peinture, pot à couleurs institutions UMR 6566 « CReAAH », Centre Jean Bérard, Soprintendenza speciale per i Beni archeologici di Napoli e Pompei, Ministère des affaires étrangères (Paris)

Index géographique : Pompéi

\section{AUTEURS}

\section{MARIE TUFFREAU-LIBRE}

UMR 6566 « CReAAH » (CNRS / Université Rennes 1), LAHM - marie-t.libre[at]orange.fr 


\section{ISABELLE BRUNIE}

LAHM (UMR 6566 / Université de Rennes 2) - isabelle.brunie[at]gmail.com

\section{SÉBASTIEN DARÉ}

Centre d'études et de recherches archéologiques du Morbihan (CERAM, Vannes) - sebastien.dare

[at]wanadoo.fr 